\title{
عروض مختصرة
}

\section{إعداد: إيصال صالح الحوامدة}

ا ـ الـراث عندـ طه عبـد الرحمن، أحمــ كروم، بيروت: المؤسسة العربية للفكر

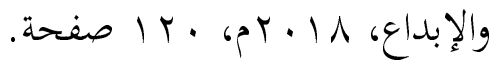

البحث في مفهوم التراث ومادته عند طه عبد الرمن بحثث في مفهوم متجذذر في

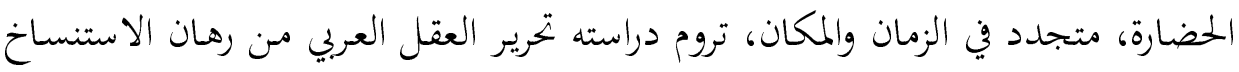

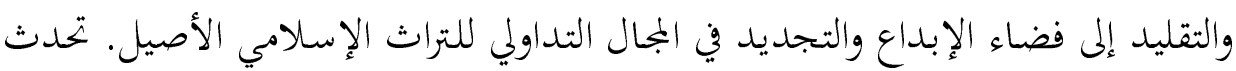
المؤلف في الفصل الأول عن مفهوم التراث وفقه آلياته في فكر طه عبد الرمن، وقارنه بين

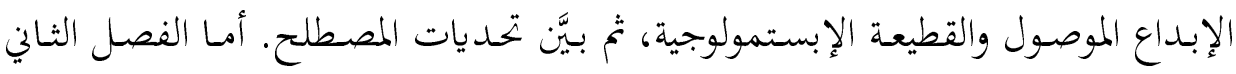

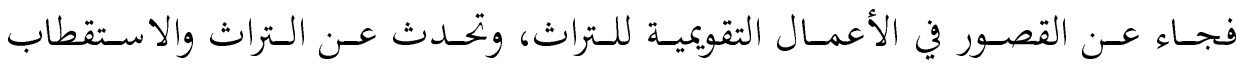

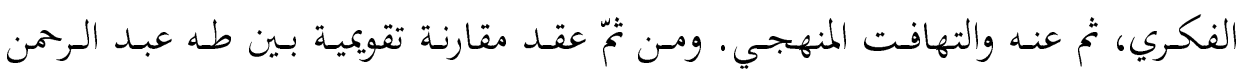

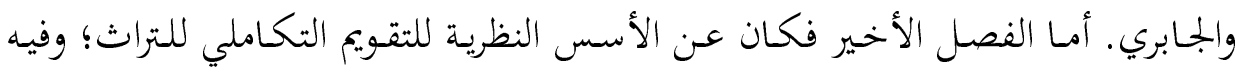

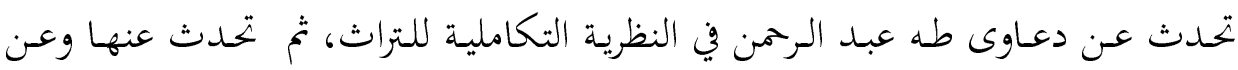
أصول التراث الإسلامي: أصل التدخل وأصل التداول، وأصل التقريب.

r. النقد الائتماني للأنموذج الدهرائ في فلسفة طه عبد الرحمن، آسيا عقوني،

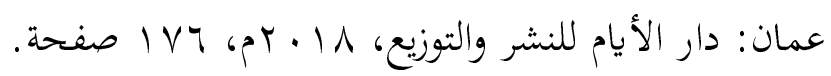

جاء الفصل الأول بعنوان: الدنيانية وميتافيزيقا الفصل؛ وفيه تكلم عن حدّ (تعريف)

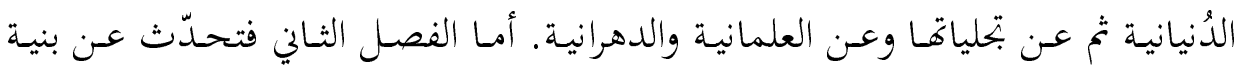

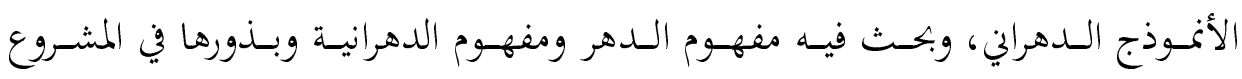

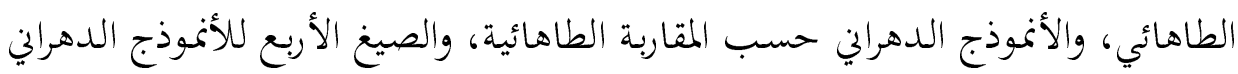

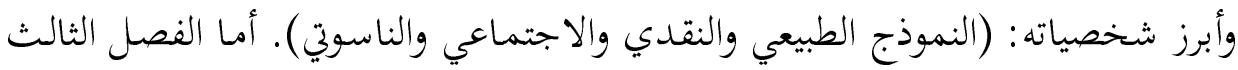

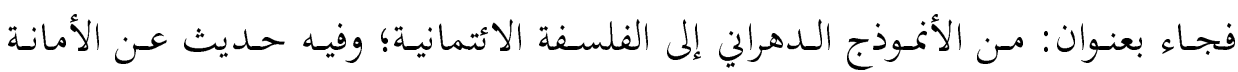


والفلسفة الائتمانية، تم عن مبادئ الفلسفة الائتمانية، تم عن الفلسفة الائتمانية في صلب الإشكاليات المعاصرة، وأخيراً مقاصد الفلسفة الائتمانية.

r. علمم الكلام الجديـد مـدخل لدراسـة اللاهوت الجمديد وجـدل العلم واللدين،

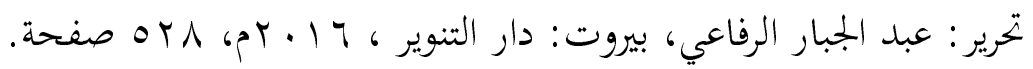

الكتاب يجوي مجموعة من الأبحاث هي: تمهيد لدراسة علم الكلام الجديد، والفلسفة

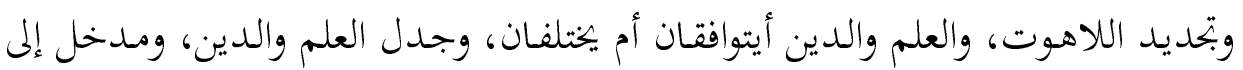

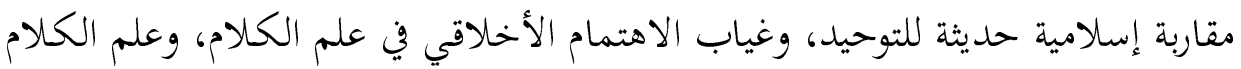

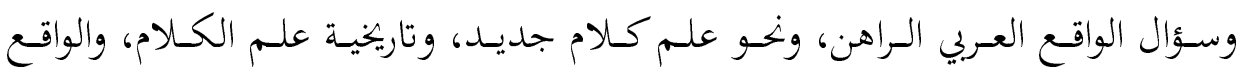

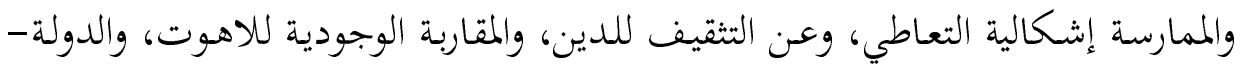

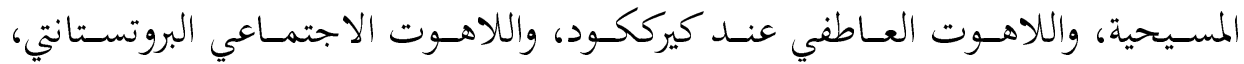
والتفكير بالله البحتث عن الحقيقة القصوى، والعقلانية وتبرير المعتقد المديني، وإشكالية الفلسفة والشريعة، وما يعد به علم الكلام الجديد، والابحاهات الجلديدة في علم الكالام. ع. المقاصـد السَّنية في بيـان القواعـد الشـرعية، عبـد الوهـاب الثـعرائ، تحقيق:

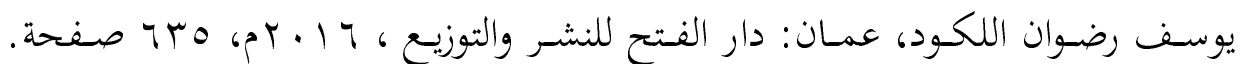

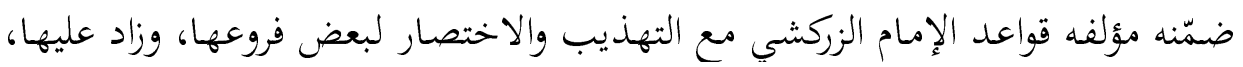

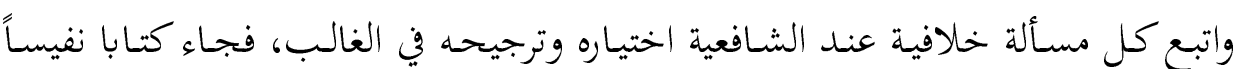
مسبوك العبارة مـن غير تطويل مـل، ولا اختصار مخلّ، بأسلوب واضحح، مرتباً للقواعد

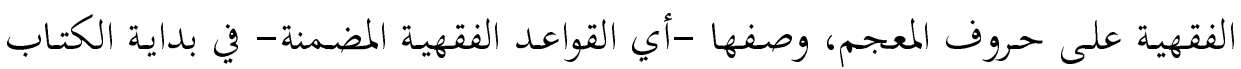
بأها قواعد عظيمة في غالب أبواب الشريعة يتعين على كل فقيه الوقوف عليها ليرجع إليها حين خوضه بالفروع، ويذكر المؤلف مسائل قاعدة في قاعدة أخرى زيادة للفائدة. هـ قاعـدة التقـديرات الشـرعية دراسـة نظريسة تأصسيلية تطبيقيسة، د. حـاتم محمــ

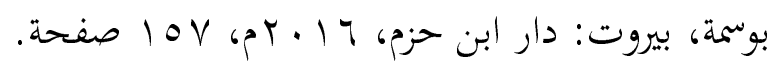
يبتـدأ المؤلــف بتعريـف القاعـدة الثـرعية، ثم في فصـله الأول يتحـــث عـن تعريـف التقديرات الشرعية وحجيتها وضوابط العمل بها، ثم في الفصل الثاني يتحدث عن علاقة 
التقديرات الشرعية بالقواعد الفقهية وأصول الفقه والمقاصد. أما الفصل الثالث فيبحث

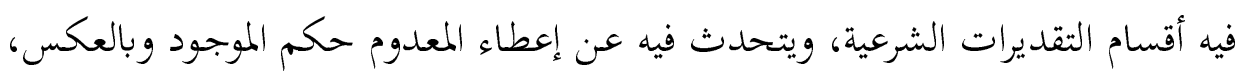

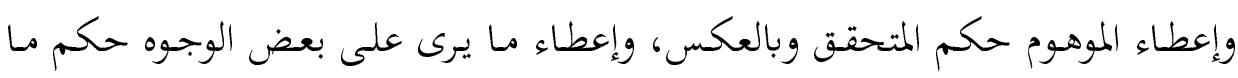
يرى على كلها وبالعكس.

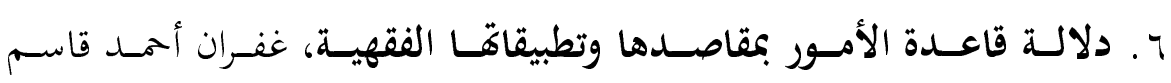

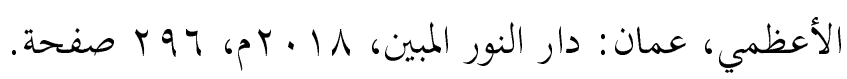
يتحدث الكتاب في الفصل الأول عن مفهوم القاعدة الفقهية ومصادرها ونشأها وأهم المصادر فيها، أما الفصل الثاني فيبحث مفهوم قاعدة "الأمور بمقاصدها"، وألهاء أهميتها

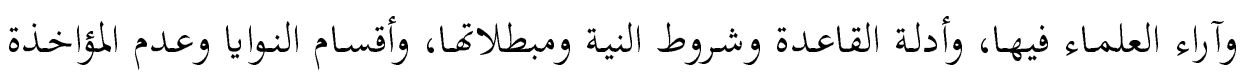

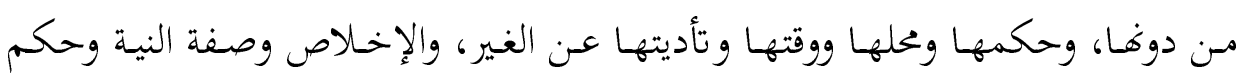

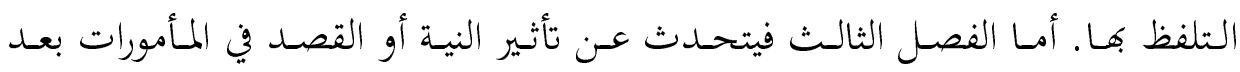

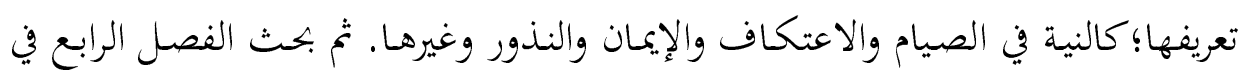

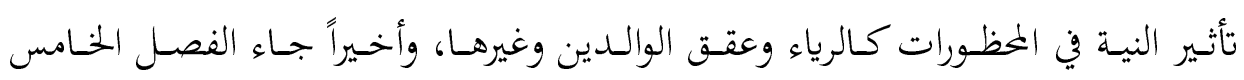

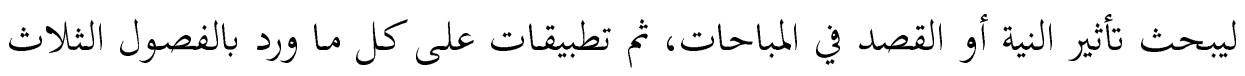
الأخيرة.

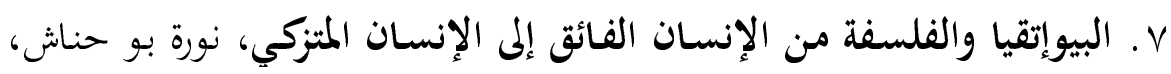

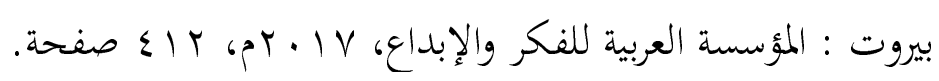

تتحـدث الباحثة في القسم الأول عن مـا بعـد الأخـاق: الخلاصدة الإبستمولوجية

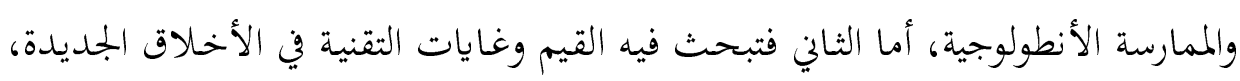

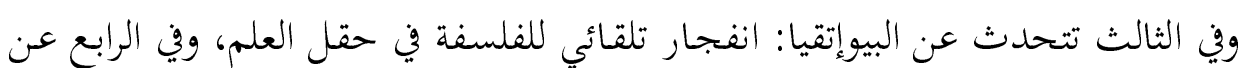

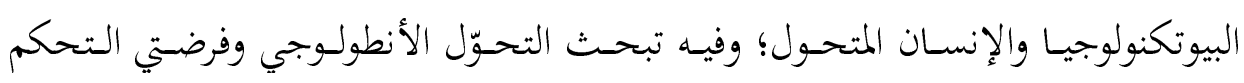

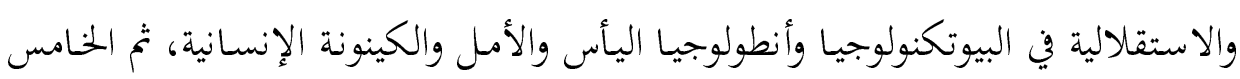

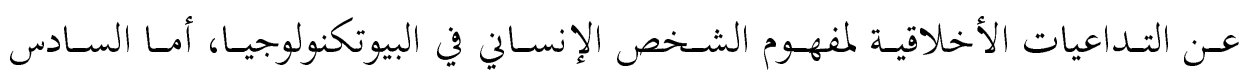


فتتحسث عسن أزمهة المعـايير والرهـانات البيوإتقيـة، وفي القسم الأخهير تتحسدث عسن الثورة الجينية وفلسفة الابتلاء في التصور الإسلامي.

^. الـدين والإنسـان والعـالم قـراءات في أفكـار إسـلامية معاصسرة، تحريـر : هبـة

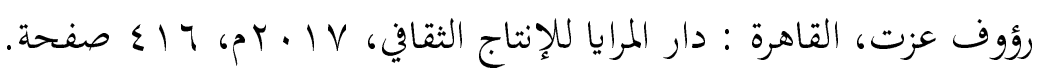
الكتـاب عبـارة عـن مجموعـة مـن البحـوث لعـدة مـؤلفين، البـاب الأول يبحــث في المفاهيم؛ إذ يتحدث فصله الأول عن مفهوم الدين، والثاني عن قراءة في تصورات الزمن

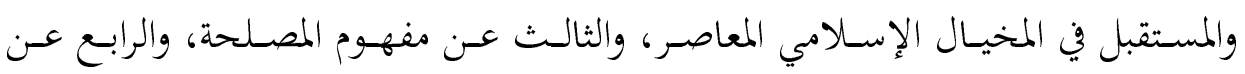
تصـورات العـدل في الفكـر الإسـلامي المعاصـر، والخـامس عـن "الإسـلاميون وإشـكالية

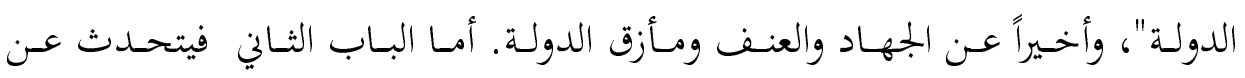
الشخصيات؛ إذ جـاء فصله الأول معنوناً بـ: استعادة التفلسف: معسالم المشروع الفكري

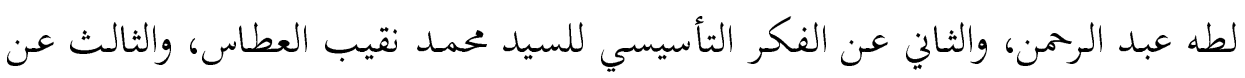
الإسلام والإنسان "أفق العالمية في فكر المسيري وبيجوفيتش"، والرابع عن وائل حلاق بين الغرب والشرق، والخامس عن مركزية الأخلاق والجمال في الإسلام: دراسة في فكر خالد أبو الفضل، والسـادس عن كليك صديقي، وأخيراً عن إنتاج مفـاهيم جديـة لإصلاح الفكر الإسلامي دراسة في مشروع طارق رمضان.

9. ظاهرة نقد الدين في الفكر الفربي الحديث، سلطان بن عبد الرحمن العميري،

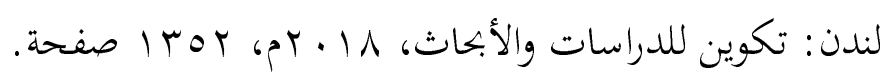
يتحـدث المؤلـف في بابـه الأول عـن مكـونات ظـاهرة نقـد الـدين في الفكـر الغربي الحديث؛ ويبحث الفصل الأول أسباب هذه الظاهرة، ويقوم الفصل الثاني بتسليط الضوء

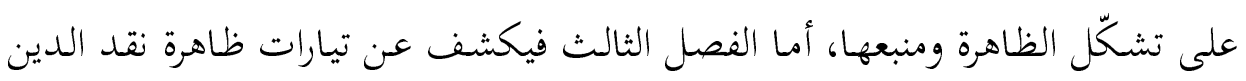

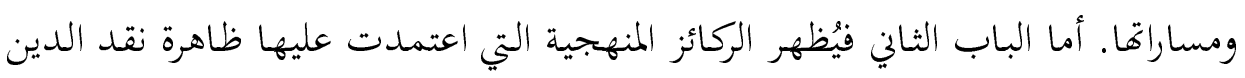
في الفكر الغربي الحمديث؛ ففي فصله الأول يبحث الركائز الفلسفية التي اعتمدت عليها 
الظاهرة، ويبحث الفصل الثاني الركائز العلمية التي اعتمدت عليها الظاهرة. ويسلط الباب الثالث الضوء على بجالات ظاهرة نقد الدين؛ فقصله الأول يبيّن الاعتراض على وجود

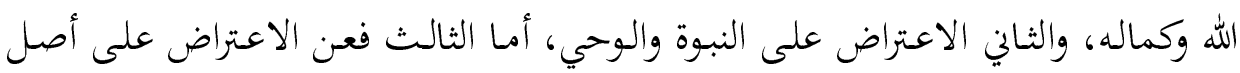
الأديان وطبيعتها.

• ا ـ الديني والدنيوي نقد الوساطة والكهنتة، عبد الإله بلقزيز، بيروت: منتدى

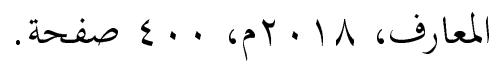
المؤلّف في القسم الأول من الكتاب يتحدث عن مقدمات نظرية في الظاهر الدينية، وفي الثناني مـداخل منهجيـة إلى دراسـة الظـاهرة الدينيـة في الموضسوعات، المفـاهيم، منـاهج

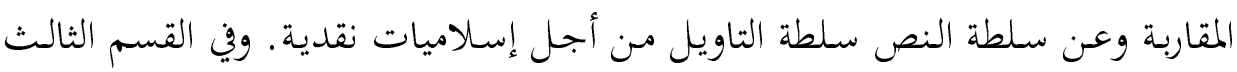

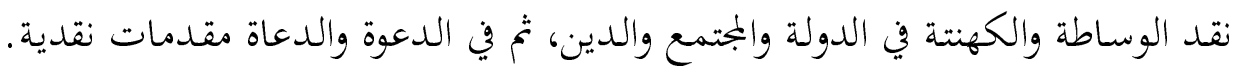

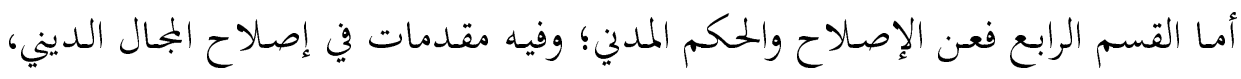
وعن الدين في الدولة الحديثة.

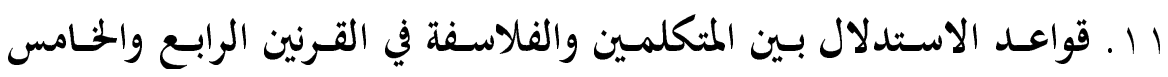

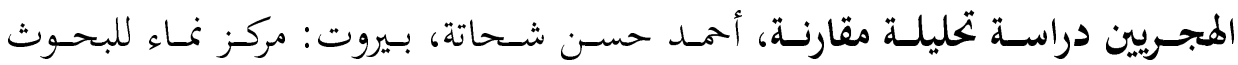

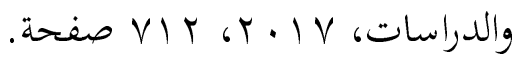

تحـدث الفصـل الأول عـن النظـر والاسـتدلال وقواعـده؛ وبحـث فيـهـ قضـاياه بــن

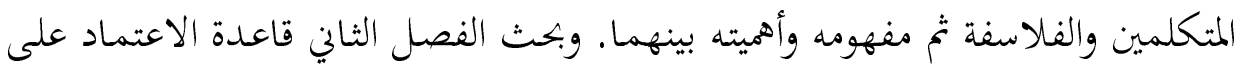

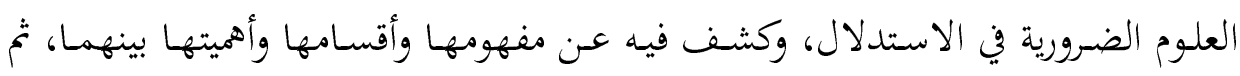

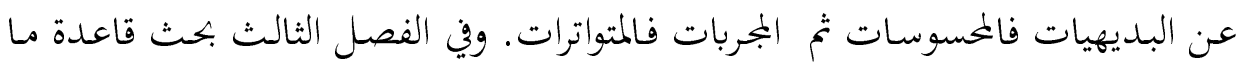

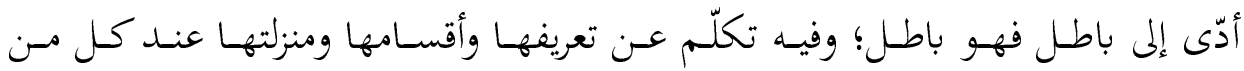

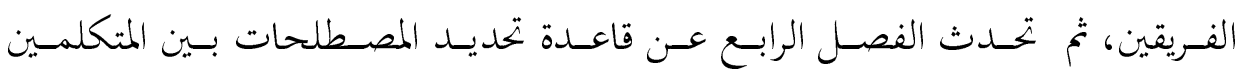
والفلاسفة؛ وفيها تعريف لها ومنزلتها عند كل منهما، ثم الحدّ بين المتكلمين والفلاسفة، فاستعمال القاعدة في الاستدلال الكامي والفلسفي. وفي الفصل الخـامس بحث قاعل قاعدة 
المعتبر في المعقـولات المعاني لا العبـارات؛ إذكشف عـن مفهومها وأهميتها والاستعمال

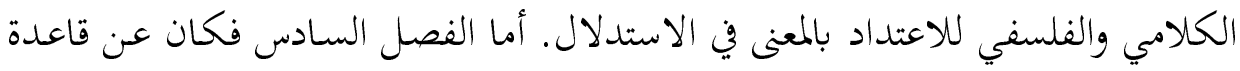
وجوب مراعاة الترتيب البنائي للأصل والفرع، وكان البحث فيه عن مفهومها واستعمالها

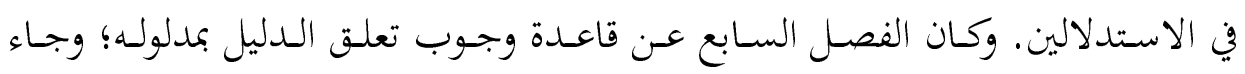
البحث فيه عن مفهومها واستعمالها في الاستدلالين.

r ا ـ نقـد العقل في الفكر العربي المعاصـر: أبـو يعرب المرزوقي أنموذجـاً، حنـان

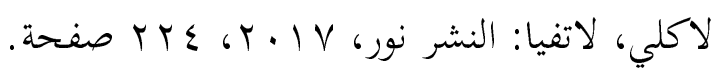

في الفصل الأول تتحدث المؤلفة عن ماهية النقد وتاريخه؛ وفيه تبحث أزمة المفهوم

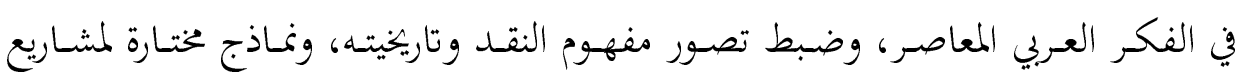

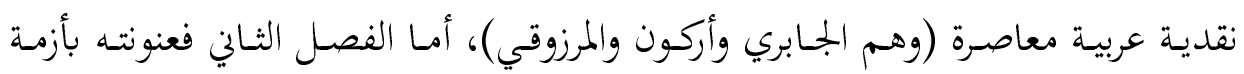

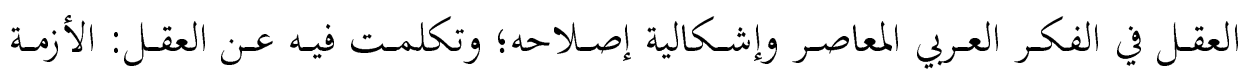

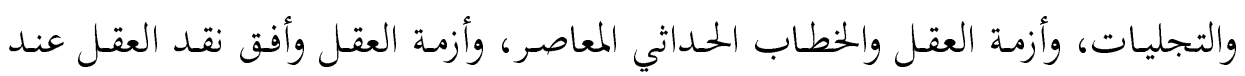
المرزوقي، ونقد النقد وإمكانية التجاوز في فكر المرزوقي. أما الفصل الثالث فكان عن نقد العقل في الفكر العربي المعاصر؛ وفيه تحدثت عن نقد النقد في الفكر العربي المعاصر بين التقويض والتأسيس، وتفريعات إصلاح العقل في الفكر العربي المعاصر، ودراسـة نقدية لطروحات المرزوقي، ومستقبل سؤال العقل والعقلانية في الفكر العربي المعاصر.

13. Sartre on Sin: Between Being and Nothingness (Oxford Theology and Religion Monographs), Kate Kirkpatrick, Oxford University Press, 2017, 262 pages.

عنسوان الكتـاب بالعربيـة: "سـارتر والخطيئسة: بـين الكينونسة والعـدم (دراسـات في

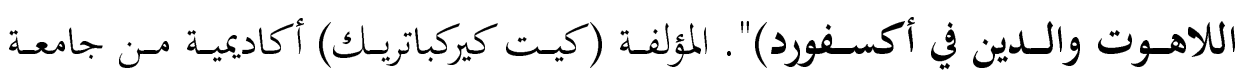
أكسفورد، متخصصة في الفلسفة واللاهوت والأدب. ترى المؤلفة أن فلسفة سارتر المبكرة المناهضة للإنسانية مدينة للعقيدة المسيحية ولفكرة الخطيئة الأصلية. 
إنّ أكثر الأفكار جوهرية وجاذبية لسارتر هي الحرية؛ إذ قدم رؤيته هذه عن طريق

الربط بين الوعي والعـدم. ترى المؤلفـة أن المناقشات الفلسفية واللاهوتيـة لأعمال سارتر افتقــت الفحص المتعمـق للتشـابه بـين القضـايا الوجوديـة لكلل مـن سـارتر وأوغسطين. وتحدَّث الكتاب عن سارتر والخنطيئة كمقدمة ثم تعرَّض لأصل العَدَم، والخطيئة بين الأنا

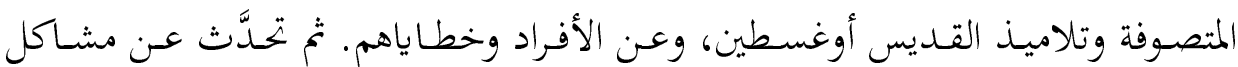
العـدم: الهويـة، والقلـق، وسـوء الإيمـان، وعـن سـقوط الـذات خـلال بحثهـا عـن الكينونـة المفقودة، وحرية الشخص في أن يكوّن لنفسه "العدم" الخناص به، ويقترح الكتاب أخيراً تطوير علم لاهوتي مسيحي خاص بالخطيئة بحسب اعتقاد سارتر، إضافة إلى موضوعات موت الله، وموت الحبب، و تأويل اليأس.

14. An Introduction to Islamic Jurisprudence, Daniel (Ghasem) Akbari , AutherHouse, 2017, 94 pages.

عنوان الكتاب بالعربية: "مدخل إلى الفقه الإسلامي"، المؤلف هو دانيال (قاسم)

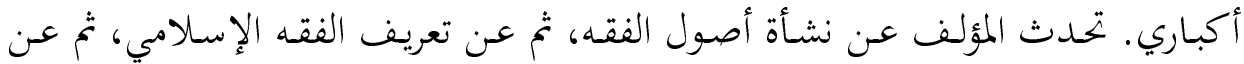

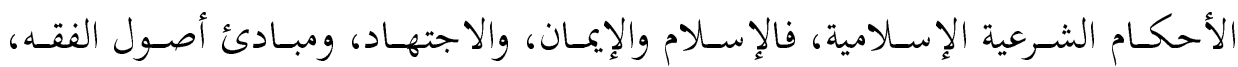
وإبطال الأحكام، ومقاصد الشريعة، وأسباب النزول.

15. Islamic Modernities in Southeast Asia: Exploring Indonesian Popular and Visual Culture (Asian Cultural Studies: Transnational and Dialogic Approaches), Leonie Schmidt, London: Rowman \& Littlefield international, 2017, 210 pages.

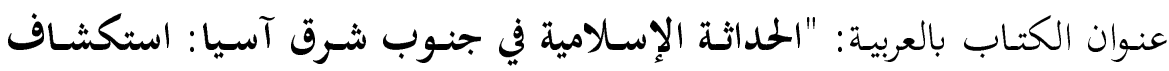

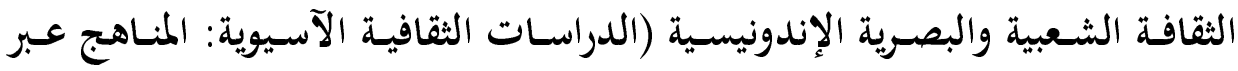
الوطنية والحوار)". المؤلف (ليون شميدت)؛ أستاذ مساعد في قسم الدراسات الإعلامية

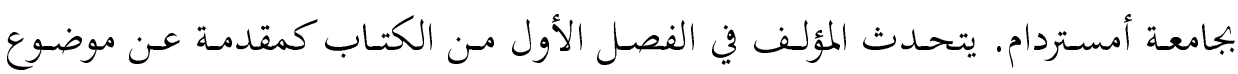

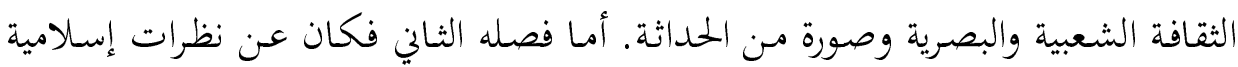


حضرية: تحويل مساحة بجمعـات التسوق خـالال رمضـان. أمـا الثالث فتنـاول موسيقى

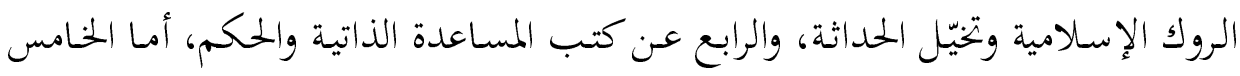

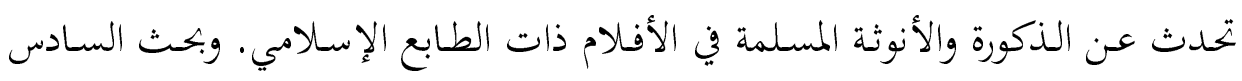

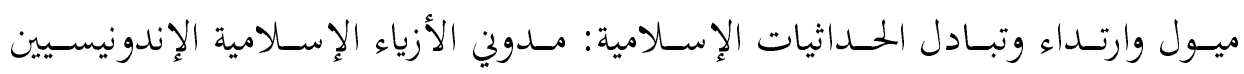
والماليزيين. وتناول الفصل السابع اكتشاف الماضي وإعادة تصور الفن المعاصر والسياسة

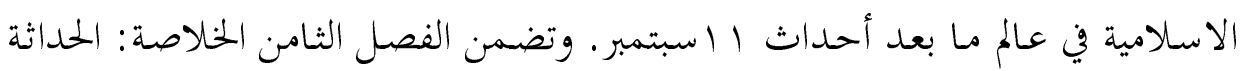
الإسلامية وسياسية التعددية.

16. The Understanding of Maqasid al-Shariah Through Usul Fiqh Mechanism, Mohd Noh \& Mohd Shahid, LAP Lambert Academic Publishing, 2016, 88 pages.

عنوان الكتاب بالعربية: "فهم مقاصد الشريعة من خلال أصول الفقه الإسلامي"، فئل

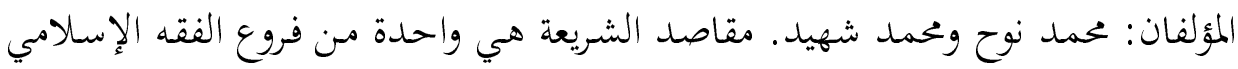

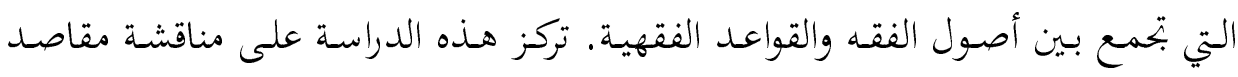

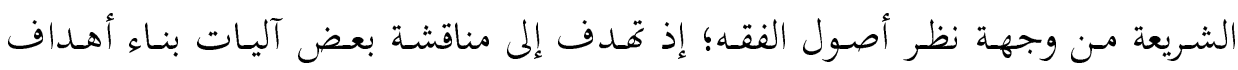

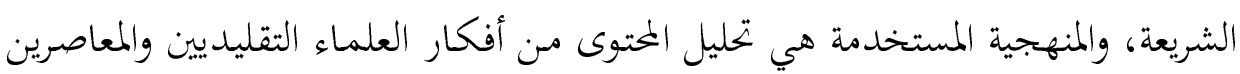

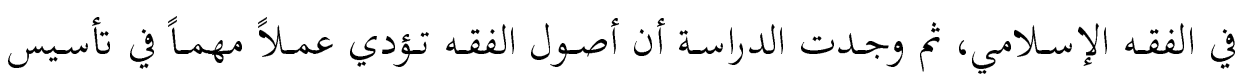

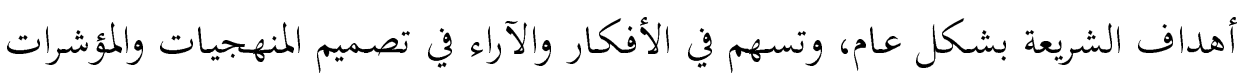
والخصائص بناءً على عدة حالات وعيّنات في الشريعة. 